\title{
Design of Control System of Manipulator based on PLC
}

\author{
Xinya Chen \\ Department of Mechanical and Electrical Engineering \\ Henan Mechanical \& Electrical Engin. College \\ Xinxiang, China \\ e-mail: xinyachen@163.com
}

\author{
Zhen Chen* \\ Department of Electronic and Communication \\ Engineering \\ Henan Mechanical \& Electrical Engin. College \\ Xinxiang, China \\ e-mail: chenzhen22@163.com \\ * Corresponding Author
}

\begin{abstract}
Manipulator is the traditional task executing agency in industrial robot system, as well as one of the key components of the robot. The mechanical structure of the manipulator consists of ball screw, slider and other mechanical devices; electric aspect consists of AC motor, inverter, sensor and other electronic devices. The device covers programmable control technology, position control technology, testing technology and the like, which is one of the typical instruments of mechatronics. The working principle of the manipulator introduced in this paper is listed as follows. Three routes of pulse output from the PLC respectively actuate the horizontal and vertical axis transducer to control the precise positioning of the horizontal and vertical axis of the manipulator, and the micro switch transmits the position signals to PLC host; position signals are fed back to PLC host by proximity switch, and the opening and closing of manipulator gripper are controlled by the positive inversion of $\mathrm{AC}$ motor, to achieve the function of manipulator's precise movement.
\end{abstract}

Keywords-Programmable logic controller;Control system;Manipulator;Output signals;Automatic program

\section{INTRODUCTION}

The schematic diagram of manipulator structure and various parts' movement is shown below. The work of manipulator is driven by motor, and its rising, declining, left moving and right moving are all completed by the rotation of the motor-driven thread screw rod.

The initial position of the manipulator stops at the origin, and the manipulator will complete a work cycle of declining, intensifying the workpiece, rising, moving right, declining, relaxing the workpiece, rising again and moving left after pressing the start button. The movement conversion of manipulator's declining, rising, moving right and moving left is controlled by corresponding limit switches, while the movement conversion of intensifying and relaxing is controlled by time.

To ensure safety, the manipulator can only decline when there is no workpiece on the right table after it moves to the right place, and it should automatically suspend and stop if the workpiece on the table has not been removed. Thus, a photoelectric switch is set to detect "no workpiece" signal.

\section{REQUIREMENTS FOR THE CONTROL}

In order to meet production requirements, manipulator is set to two modes of manual working mode and automatic working mode, and automatic working mode is further divided into single-step, single-cycle and continuous working mode.

\section{A. Manual Working Mode}

Using buttons to control every movement of the manipulator. For example, the manipulator would decline when "decline" button is pressed; the manipulator would rise when "rise" button is pressed. Manual operation can be used to adjust the working position and to control the manipulator to return to the origin after an emergency stop.

\section{B. Single-step Working Mode}

From the origin and in accordance with the step sequence of automatic working cycle, the manipulator stops automatically after the completion of a movement each time the start button is pressed.

\section{Single-cycle Working Mode}

When the start button is pressed, the manipulator automatically completes a cycle of movement in accordance with procedure and stops after returning to the origin.

\section{Continuous Working Mode}

When the button is pressed, the manipulator automatically, repeatedly and continuously works from the origin according to the step sequence. And two parking states under continuous working mode are set. Normal Parking: parking under normal working condition. The manipulator returns to the origin and automatically shuts down after completing the last cycle, when the reset button is pressed. Emergency parking: parking in the event of an accident or emergency. The manipulator stops at the current state, when the emergency stop button is pressed. When trouble is removed, it can only return to the origin manually.

\section{DESIGN OF HARDWARE SYSTEM}

The hardware system design includes the design of mechanical part and electric control part.

\section{A. Structure of Manipulator}

Design of structure is as shown in Fig .1. 


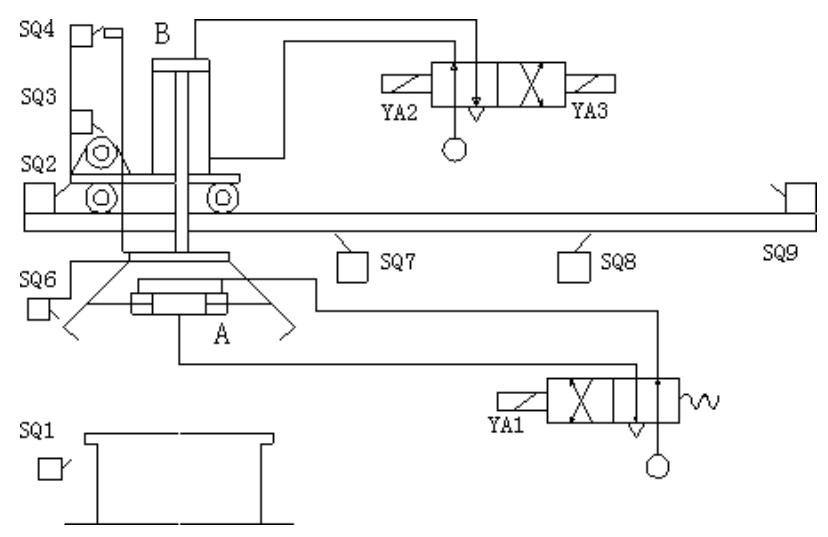

Figure1.Schematic diagram of the structure of manipulator

Setting nine travel switch SQ1-SQ9 for detection of parts, car, the position and the mechanical manipulator gripper clamping, relaxed state, and the implementation of system control. Where SQ1 for the work of the detection switch is in place, SQ2 for the car in situ detection switch; SQ4, SQ3 respectively for the mechanical hand down rise is the detection switch; SQ6, SQ5 respectively for mechanical hand clamping and relaxation detection switch; SQ8, SQ7 respectively for car speed conversion switch; SQ9 for small car movement stop switch.

\section{B. Design of Electrical Control}

The design of electrical control includes the design of the main circuit and control circuit. Main circuit composed of two motors, namely the slow motor and fast motor, respectively, dragging car slow and fast, its control are as follows: slow motor M1 by contactors KM1, km2 respectively control the Gump and inversion; fast motor M2 is composed of a contactor KM3 and $\mathrm{km} 4$ respectively control the Gump and reverse. The clamping and loosening action of the mechanical hand is completed by a hydraulic cylinder controlled by a single electric two position four way electromagnetic valve. In the case of power supply, the mechanical hand is loosened, and when the power is released, the mechanical hand caused by the sudden power failure during the operation of the equipment can be prevented.

\section{Operation Panel and Action Description}

According to the requirements of control and production process, the control operation includes manual and automatic, manual and manual steps, back in situ operation, automatic control including single step, single cycle, continuous operation. So the operation mode selection switch is set with five stalls. Manual mode, the manual operation including up, down, relax, fast forward, slow progress, rewind, slow back and reset, so set the six movements reader button. All movements are carried out with the action indicator light. In addition, a start stop button is provided.

\section{I/O distribution}

$\mathrm{I} / \mathrm{O}$ equipment that is required for the $\mathrm{I} / \mathrm{O}$ points as shown in the following table.

TABLEI. I/O POINT ALLOCATION

\begin{tabular}{|c|c|c|c|c|c|}
\hline signal & $\begin{array}{l}\text { I/O } \\
\text { Equipment }\end{array}$ & $\begin{array}{l}\text { I/O } \\
\text { Points }\end{array}$ & signal & $\begin{array}{l}\text { I/O } \\
\text { Equipment }\end{array}$ & $\begin{array}{l}\text { I/O } \\
\text { Points }\end{array}$ \\
\hline & mode choice & 5 & & Control coil & 4 \\
\hline & Manual & 8 & & Solenoid & 3 \\
\hline \multirow{3}{*}{ Input } & movement & & \multirow{3}{*}{ Output } & valve & \\
\hline & Start/Stop & 2 & & $\begin{array}{l}\text { Action } \\
\text { indication }\end{array}$ & 8 \\
\hline & travel switch & 9 & & $\begin{array}{l}\text { Origin } \\
\text { indication }\end{array}$ & 1 \\
\hline
\end{tabular}

According to the distribution of I / $\mathrm{O}$ requirements and consider the I / O allowance of $10 \%$ to $15 \%$, the design of PLC with F1 - 60MR 36/24 type and layout is shown in Fig..

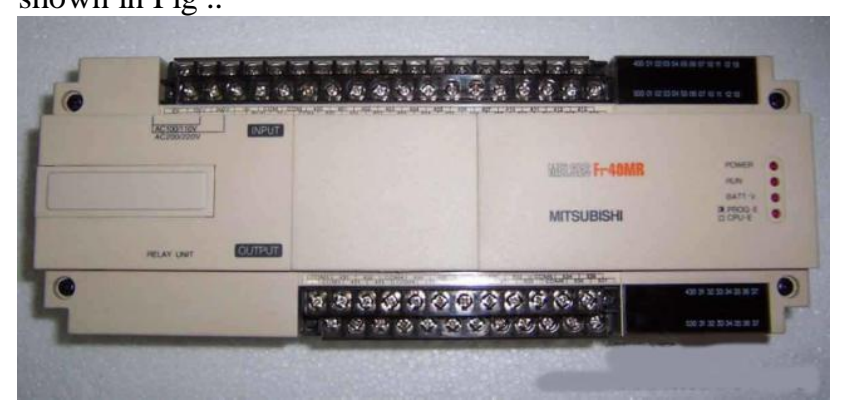

Figure2.F1-40MR prototype

\section{PLC CONTROL SYSTEM}

\section{A. Determining the Input / Output Points and Selecting PLC Model}

\section{1) Input signals}

Position detection signals: lower limit, upper limit, right limit and left limit, requiring four input terminals. "No workpiece detection" signal: photoelectric switch is applied as detecting element, requiring one terminal. "Working mode" selective switches: manual, single-step, single-cycle, and continuous working mode, requiring four output terminals. Manual operation: declining, rising, right moving, left moving, intensifying, and relaxing buttons are required, and six input terminals are also required. Auto operation: yet to start, normal stop and emergency stop buttons are required, and three input terminals are also required. Thus, a total of 18 input signals are required..

\section{2) Output signals}

The output of PLC is used to control the declining, rising, right moving, left moving, intensifying and relaxing of manipulator, the rotation speed of three motors and so on, requiring a total of 11 output points. The manipulator starts working from the origin, requiring an origin indicator, and also requiring an output point. So, at least six output points are needed.

Since the control of manipulator belongs to switch controlling, no special request is proposed for the function, and any type of small PLC can meet the requirements. Depending on the required I / $\mathrm{O}$ total points and leaving some reserved points, FX2N-48RM can be used, which has 24 input points and 24 output points respectively and is relay output type.

\section{B. PLC Control System Programming}

In order to facilitate programming, relatively independent program segments are respectively compiled for manual and automatic programs, and jump instruction is applied to select and control the structure diagram of the system program. When manual mode is selected, X3 is connected, bypassing the automatic program and performing the manual program; when automatic working mode is selected, X3 is disconnected, performing the automatic program.. 


\section{1) General part of ladder diagram}

a) State initialization: The initialization state device S600 is set and reset in the manual mode. When the switch is placed in the "return in situ" (X514 connection), press the reset button (X507) when the set, in the "manual operation" (X510) on, S600 reset. In the middle of the state machine with manual reset operation, that is, in the way to select the switch is located in the "manual operation" or "return in situ", the intermediate state machine synchronization reset. So the initialization ladder diagram is shown in Figure 3, (if the state machine starts to continue working without the condition of the power supply when the power supply is supplied, it does not need M71).

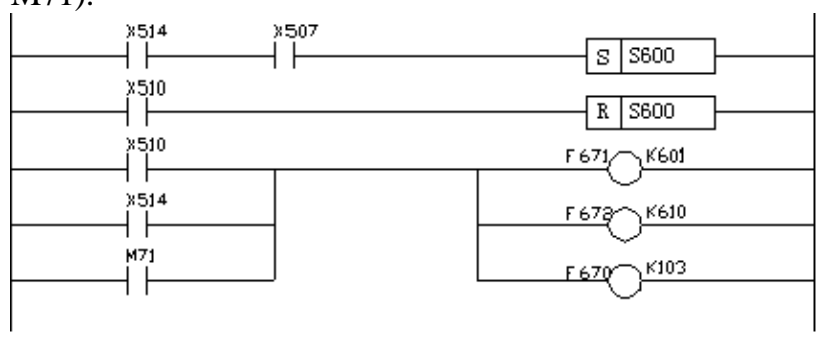

Figure 3.State initialization ladder diagram

b) State switch boot:If the manipulator works in the automatic operation mode, when the initial state device S600 is set to press the start button, the auxiliary relay M575 work, the state of the state can be a step by step downward transfer, you can carry on the conversion. In the implementation of the "continuous process", the conversion of the relay M575 has been maintained until the shutdown button down. On the other side, M100 is used to check whether the machine is in the position. When both M575 and M100 are switched on, the initial state of the device is switched on, so the ladder diagram is shown in Fig .4.

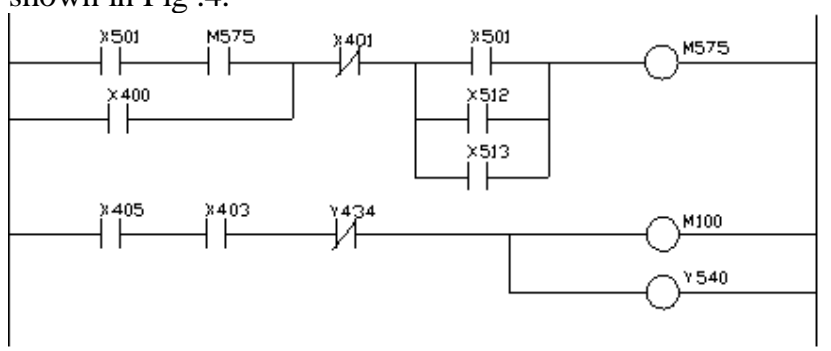

Figure 4.State converter start ladder diagram

c) State converter ban ladder diagram:

When the M574 of the special auxiliary relay is activated and the state switch is converted by step ladder instruction, the conversion of the state machine is automatically forbidden. During the "single cycle" operation, when the stop button is pressed, the M574 should be energized and maintained, and the operation is stopped at the current step. When you press the stop button, from the existing work step to start work, M574 should be reset, that is, to allow the new conversion. In the "step" mode of operation, M574 should always work, at this time, to prohibit any state transition. But did not press the start button once, M574 off once, allow the state to switch once. State transitions are prohibited in the "manual" mode of operation. After the manual mode is released, press the start button, then the state transition is forbidden, and the M574 is reset..

When the PLC is started, the M71 and M574 are used to hold the initialization pulse, and the state is forbidden to be converted until the start button is pressed. So the state converter is forbidden to ladder diagram as shown in Figure 5

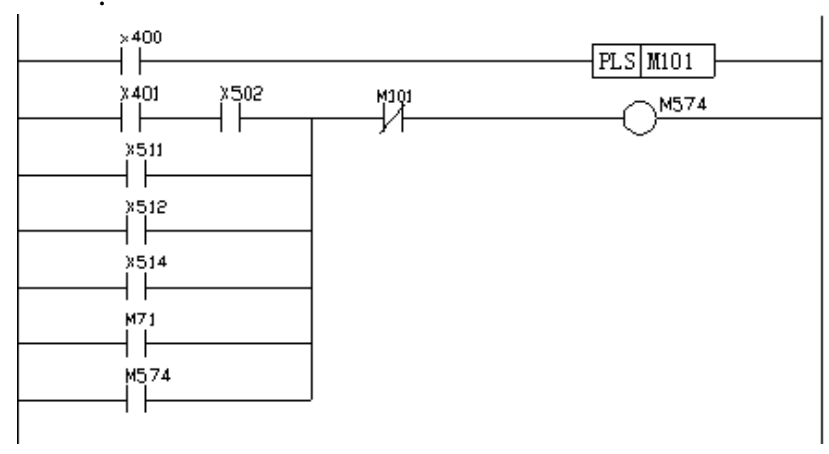

Figure 5.State converter ban ladder diagram

\section{2) Manual Program}

Manual operation need not to be conducted according to the step sequence, so it can be designed in accordance with ordinary relay program. Ladder diagram of manual operation is shown in Fig . manual buttons X20-X25 respectively control the movements of declining, rising, right moving, left moving, intensifying and relaxing. To ensure the safe operation of the system, some necessary chains are set up. X11 is added to the circuit of left and right moving as upper limit chain, because the manipulator is allowed to move left and right only when it is at the upper limit position.

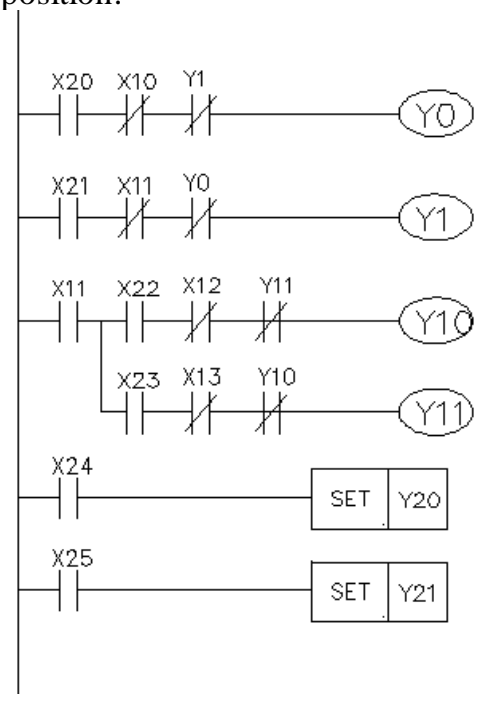

Figure 6.Manual program

3) Automatic ProgramContinuous and single-cycle operation.:

When the manipulator is at the origin, the program is in the initial state S0, and performs the movement of declining. When it lowers to the limit switch, X10 is connected, and turns on the next state S21, then performing the next movement. After the last step of movement, the manipulator moving left and touching the left limit switch, is executed, X13 is connected. If it is a single-cycle operation, M0 is disconnected and backs to 
the initial state; if it is a continuous operation, M0 is connected, the state transfers to S20, and the cycle of next period starts.

In operation, if the normal stop button is pressed, X1 is connected, M0 is reset, and the movement of manipulator completes one cycle and backs to the initial state. If the emergency stop button is pressed, X2 is connected, state S0 $\sim$ S33 are all reset, and the manipulator stops working. When it restarts, the manipulator is first moved to the origin by human hand and the can conduct automatic operation again.

Single-step operation When the design of automatic operating program applies step instruction, single-step operating program is realized by applying "prohibiting state transfer" markers M8040, as is shown in Fig. When the relay coil is turned on, the step state transfer is prohibited, and when the coil is turned off, the step state transfer is allowed.

Under the single-step operation mode, start button X0 is used as a single-step operation signal, and X4 is turned on. When the start button is not pressed, $\mathrm{X} 0$ is disconnected, its normally closed contact is closed, M8040 is turned on, and the state transfer is prohibited.

After a step of movement is completed, the start button is pressed, X0 is turned on, its normally closed contact makes M8040 disconnected, and the state transfers to the next step. Connecting the ladder diagram of single-step operation to the upper end of the automatic program can obtain the entire ladder diagram of automatic operation including single-step, single-cycle and continuous operation.

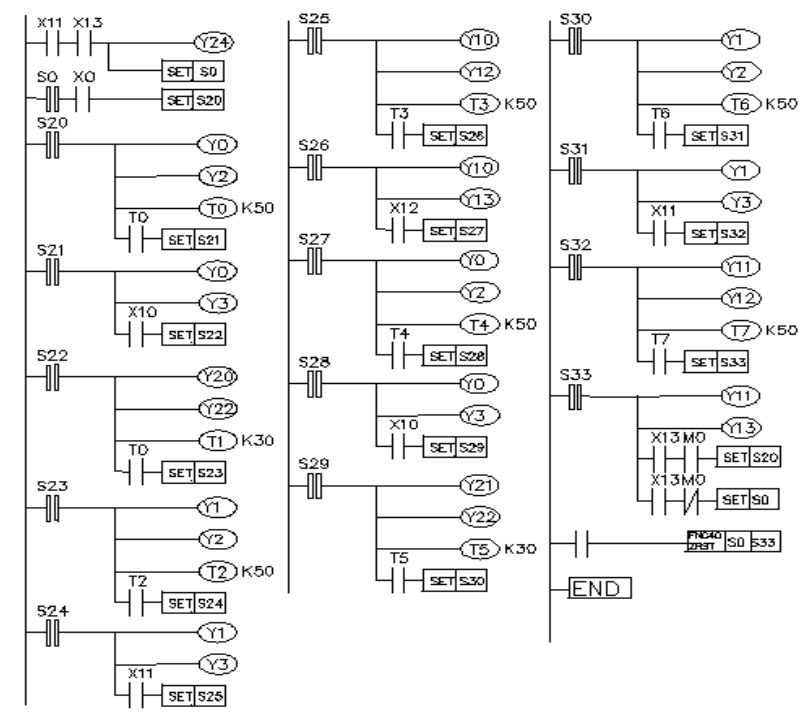

Figure 7. Automatic Program

\section{CONCLUSIONS}

This paper intends to develop materials handling manipulator, and applies PLC of FX2N series from Japan's Mitsubishi Corporation to control the rising, declining, right moving, left moving and grabbing motion of the manipulator. The mechanical structure of the device consists of ball screw, slider, mechanical gripper and the like; electric aspect consists of AC motor, inverter, console and other components. Programmable technology and corresponding hardware devices are applied to control the manipulator to complete a variety of actions.

\section{REFERENCES}

[1] CHEN Yong li,LI Li, "Design of Control System of Manipulator based on PLC and Stepping Motor," Journal of Anyang Normal University, vol.10,pp.45-52,April ,2013.

[2] YQ Wang,"Control system design of the manipulator based on PLC,"Chinese Hydraulics \& Pneumatics,vol.6,pp.104 113,February,2011.

[3] GUO Yan ping,ZHANG Chao ying, "Control System of Industrial Manipulator Based on PLC", Instrument Technique \& Sensor,vol.33 pp.68-73,October,2013.

[4] LI Ming,LI Quanqing, "Design of the Hydraulic Conveying Manipulator Based on PLC," Machine Tool \& Hydraulics, vol.53,pp. 113-122,May,2014.

[5] W Hu,L Guo, "Research of Automation Control System of Manipulator Based on PLC,"Advances in Future Computer and Control Systems,vol.67, pp.39-47,June,2012.

[6] XM Sheng,L Liu, "Automatic Placing and Removing Manipulator of the Workpiece on Stretcher Based on Electric-Pneumatic Control and PLC Control," Applied Mechanics \& Materials, vol. 2, pp. 740-741, August ,2011.

[7] T Yao,ZY Chang, "Kinematics Model of the 6-DOF Underwater Manipulator and Control System Design Based on PLC Technology," Applied Mechanics \& Materials, vol. 37, pp. 40-51, 2014.

[8] J Qin,ZL Jin, "Design of Two-Axis Manipulator System Based on PLC," Techniques of Automation \& Applications, vol. 12, pp. 99107, 2014.

[9] HU Zhigang,"Control System Design of the Pneumatic Manipulator Based on S7-200 PLC,'Mechanical Engineer,vol. 6, pp.30-41, 2014.

[10] TP Liu, J Liao, "Electrical Control System Design of Five-axis Manipulator Based on Double PLC," Techniques of Automation \& Applications, vol. 42, pp. 57-64,Dec. 2013. 